\title{
Intrapalpebral Extending Dysesthetic Bleb Revision with Fibrin Glue
}

\author{
David P Holmes ${ }^{1}$, David K Manning ${ }^{2}$
}

\begin{abstract}
Aim: This pilot study evaluates the safety and efficacy of a novel surgical technique using fibrin glue to treat bleb dysesthesia post-trabeculectomy due to intrapalpebral extension.

Background: Trabeculectomy remains the gold standard for control of intraocular pressure (IOP) in refractory glaucoma. Bleb dysesthesia following antifibrotic-enhanced trabeculectomy is common, resulting in a significant decrease in quality of life. Symptoms include pain, foreign body sensation, and excessive tearing. Treatments include lubrication, topical nonsteroidal anti-inflammatory drugs (NSAIDs), bandage contact lens, bleb needling, compression sutures, Nd:YAG laser treatments, autologous blood injection, and cryopexy. These procedures can be timeconsuming, risk bleb function, and may be ineffective at symptom control; thus, a novel technique is required.

Technique: This is a retrospective case series of eyes undergoing bleb dissection with scleral cutdown and conjunctival closure with ARTISS fibrin tissue glue. Reported cases have been followed up for 6 months. Outcomes assessed include complications, IOP, medication usage, subjective pain score, visual field, and cup-to-disk (C:D) ratio.

Conclusion: This small pilot series demonstrated that patients suffering from bleb dysesthesia due to intrapalpebral bleb extension can be successfully treated with a novel surgical approach combining conjunctival dissection to sclera with fibrin tissue glue closure. There was no significant effect on bleb function and no contribution to glaucoma progression over a 6-month period. A significant reduction in patient discomfort with no new surgical complications was noted.

Clinical significance: This demonstrates a safe and effective novel surgical approach to treat patients with this condition. The technique is easily learnt and can be employed in an outpatient setting. The technique is readily accepted by patients in group who are often reticent to undergo further intervention. Most importantly, it does not compromise bleb function or destabilize glaucoma control.

Keywords: Bleb, Dysesthesia, Fibrin glue, Glaucoma, Intraocular pressure, Surgical technique, Trabeculectomy.

Journal of Current Glaucoma Practice (2020): 10.5005/jp-journals-10078-1270
\end{abstract}

\section{BACKGROUND}

Trabeculectomy remains the gold standard for the control of intraocular pressure (IOP) in refractory glaucoma. It involves the creation of a controlled subconjunctival drainage area for the aqueous known as a bleb. With the introduction of antimetabolites intraoperatively, the long-term outcomes for trabeculectomy have improved. These antimetabolites, such as mitomycin C (MMC) and 5-fluorouracil (5-FU), promote the development of diffuse, thinner walled, less vascular blebs which are beneficial for IOP control and the prevention of bleb failure. ${ }^{1,2} \mathrm{~A}$ Cochrane review found that MMC has a slight advantage in incidence of trabeculectomy failure, IOP lowering effect, and epitheliopathy. However, MMC was associated with increased risk of bleb and wound leaks, late hypotony, and cataract formation. ${ }^{3}$ However, the development of bleb dysesthesia has also increased as a result, particularly with bleb extension into the intrapalpebral fissure. The feeling of dry eye and ocular irritation may also be further potentiated by irregular surface epithelium, a largely avascular and acellular subepithelium, and decreased goblet cells, as demonstrated in studies of the histology of blebs created from MMC augmented trabeculectomy. ${ }^{4}$

Bleb dysesthesia is a syndrome caused by the bleb interfering with normal eyelid function and tear film distribution. The symptoms may include ocular pain, discomfort, burning, foreign body sensation, and excessive tearing. The experience of pain is significantly governed by psychological factors. It has been shown that chronic pain exacerbates anxiety which then further potentiates the experience of pain. This means that patient satisfaction with a surgical intervention aimed at reducing bleb dysesthesia would be
${ }^{1}$ Department of Ophthalmology, The Royal Victorian Eye and Ear Hospital, East Melbourne, Australia

${ }^{2}$ Department of Ophthalmology, Hunter Cataract and Eye Centre, Charlestown, Australia

Corresponding Author: David P Holmes, Department of Ophthalmology, The Royal Victorian Eye and Ear Hospital, East Melbourne, Australia, Phone: +61 437739410, e-mail: holmesdp93@ gmail.com

How to cite this article: Holmes DP, Manning DK. Intrapalpebral Extending Dysesthetic Bleb Revision with Fibrin Glue. J Curr Glaucoma Pract 2020;14(1):37-42.

Source of support: Nil

Conflict of interest: None

dependent on a decrease in their experience of pain and irritation more so than any objectively measurable outcome. ${ }^{4,5}$

The development of bleb dysesthesia is influenced by a number of factors. Young age, superonasal bleb location, poor lid coverage, and a prominent bleb with bubble formation have all been associated with bleb dysesthesia. Additional factors include steep walls, elevation of blebs, or significant posterior or intrapalpebral extension. ${ }^{4-6}$

Bleb revision surgery has been described in a number of articles and a number of different techniques; however, these mostly deal with trying to increase drainage of under-filtering blebs or decrease drainage of over-filtering or leaking blebs. There have been some techniques reported for managing bleb dysesthesia. ${ }^{6-8}$ 
Treatments available for treatment of bleb dysesthesia include lubrication, topical nonsteroidal anti-inflammatory drugs (NSAIDs), bandage contact lenses, bleb needling, compression sutures such as the Palmberg and modified checkerboard techniques, Nd:YAG laser treatments, autologous blood injection, and cryopexy, all of which have modest degrees of success and are associated with complications including ongoing bleb dysesthesia, increase in IOP, and subsequent failure of the bleb. ${ }^{5,7,9,10}$

In cases of refractory bleb dysesthesia, there are four main methods available: autologous conjunctival bleb resurfacing (ACBR), bleb reduction, conjunctival advancement with ologen collagen matrix implant, and bleb excision with closure of the fistula and insertion of a drainage implant. Eyelid lowering procedures have also been reported to decrease the sensation of dysesthesia. ${ }^{6,11}$

Each has disadvantages including reported increases in postoperative IOP. There are also specific risks for each procedure. In ACBR, the loss of conjunctiva from the graft harvest, usually inferiorly, means that if the bleb were to subsequently fail, it may be more difficult to place a tube. The primary disadvantage of conjunctival advancement is the potential for bleb leak and additional scarring can lead to bleb failure, so care also needs to be taken to avoid creating a buttonhole. ${ }^{12}$ As with any conjunctival advancing procedure, it can also induce a slight ptosis. Excision of the bleb requires another drainage procedure. ${ }^{12}$

An effective and reliable method for improving the symptoms of bleb dysesthesia reliably without compromising bleb function, creating a leaking bleb, or developing possible endophthalmitis as a consequence is needed, ideally without the added irritation and inflammation associated with sutures. ${ }^{7}$

Fibrin glue, which contains two human derived plasma products, fibrinogen with a synthetic fibrinolysis inhibitor and thrombin, such as ARTISS manufactured by Baxter Pharmaceuticals, is currently regularly used in pterygium surgery and conjunctival interventions for conjunctival closure. In glaucoma, it is commonly used in association with tube surgery with reported benefits of increased early postoperative comfort and a reduced inflammatory response. It is very effective in creating a watertight seal between tissue edges due to the wide field effect of appositional glue closure as opposed to intermittent localized compression from suturing. This enables a conjunctival incisional procedure to be completed without the need for sutures, with minimal postoperative irritation and a low risk of bleb leakage. ${ }^{13}$

The purpose of this pilot study is to evaluate the safety and efficacy of a novel surgical technique using fibrin glue to treat bleb dysesthesia post-trabeculectomy due to intrapalpebral bleb extension. It is presumed that the use of fibrin glue will enable successful barrier formation to reduce bleb size and improve ocular comfort, yet cause minimal postoperative distress in a group of already dissatisfied patients.

\section{TECHNIQUe}

Patients in this study were identified as having significant selfreported bleb dysesthesia, in the setting of a functional bleb without hypotony. In general, the subjects had significant bleb extension into the intrapalpebral fissure, with normal bleb morphology. All patients had been unsuccessful with conservative therapies such as intensive lubricant use.

Patients' pain levels were assessed using a subjective pain score. A 0 to 10 unilinear numeric pain grading scale (Fig. 1) was administered by clinical staff. The tool was used at both the preoperative and 6-month postoperative consultations.

Objective bleb measurement was performed pre- and postoperatively using the standard reference images from the Indiana Bleb Grading Scale (Fig. 2). ${ }^{15}$ A single observer undertook the examination, and bleb morphology was assessed with regard to bleb height, extent, vascularity, and leakage.

Patients were brought to the operating theater after undergoing full informed consent. All patients underwent local anesthetic peribulbar injection with $2 \%$ lignocaine, combined with sedation. Betadine preparation was performed followed by Mellinger speculum placement.

A 7-0 vicryl corneal stay suture was placed in the peripheral superior cornea, with the eye infraducted and held in place with an artery forceps to expose the trabeculectomy bleb in the superior bulbar conjunctiva (Fig. 3).

The relative lid position was noted, and targeted conjunctival marking was undertaken to identify the proposed position of conjunctival incisions, either side of the bleb, ensuring that they were placed beyond the palpebral fissure and also to enable easy identification of the cut conjunctival edge to ensure accurate repositioning at the surgery conclusion.

Radial incisions were made with Westcott scissors down to sclera, from the limbus to the highest visible portion of the bulbar conjunctiva. Undermining of the conjunctiva was performed to $2 \mathrm{~mm}$ beyond the cut edge away from the bleb (Fig. 4). ARTISS was decanted into its two separate components, with the thicker solution initially placed within the wound and subsequently activated by the less viscous portion. A separate application was employed to control the volume of the solution used and to control the timing of activation (Fig. 5).

The cut conjunctival edges were reopposed, ensuring adequate tissue glue adhesion to the globe below the undermined area, as well as direct adhesion of the two incised edges, pinched together over the whole incision length with Moorefield's forceps (Figs 6 and 7).

Removal of the stay suture and withdrawal of the speculum occurred once the glue was cured at 3 minutes. Patients were administered antibiotic eye drops (tobramycin) for 1 week and prednisolone eye drops postoperatively for 3 weeks on a tapering

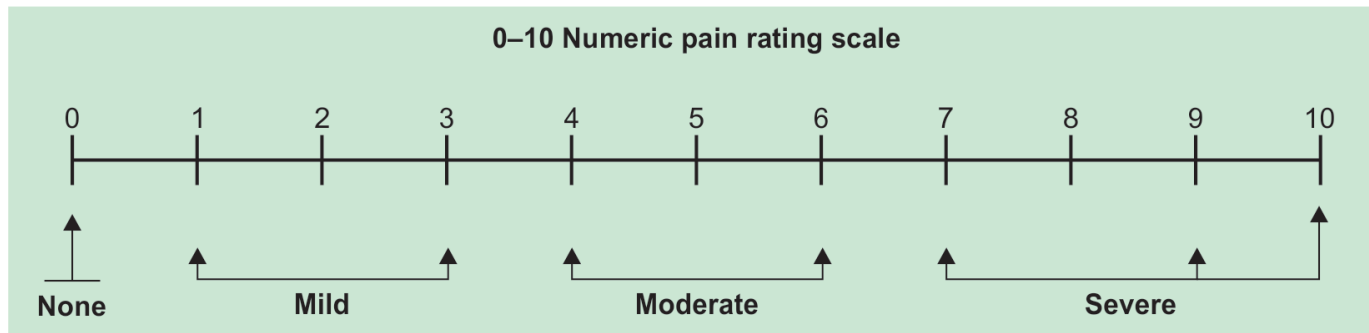

Fig. 1: Unilinear numerical pain rating scale ${ }^{14}$ 


\section{Bleb height}

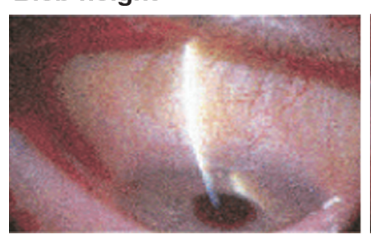

HO: Flat bleb

Horizontal extent

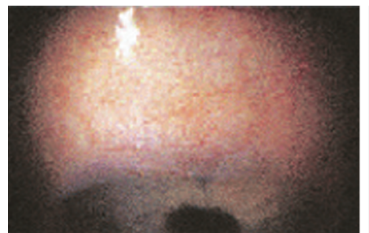

EO: $0<1$ clock hours

Vascularity

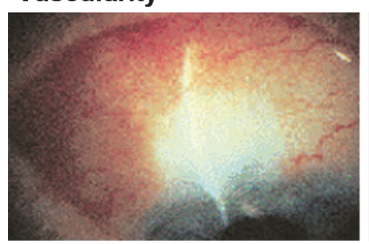

V0: Avascular white

Seidel test

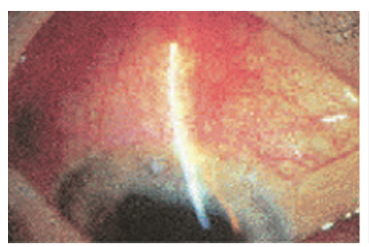

V4: Extensive vascularity

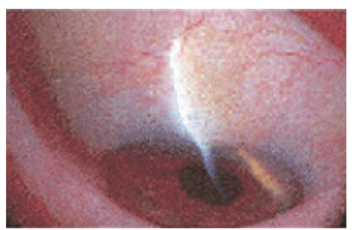

H1: Low bleb

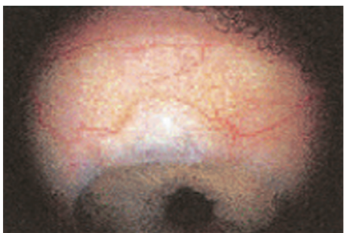

E1: 1-2 clock hours

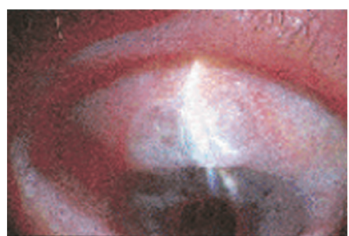

V1: Avascular cystic

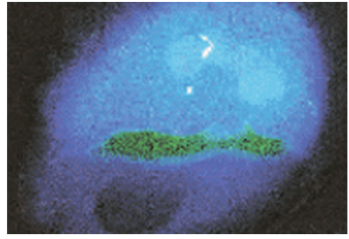

S0: No leak

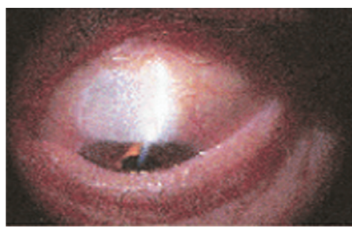

H2: Medium bleb

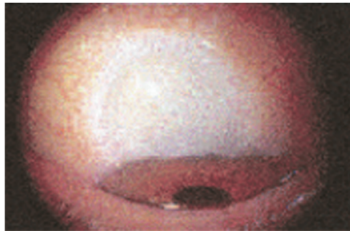

E3: $>2-<4$ clock hours

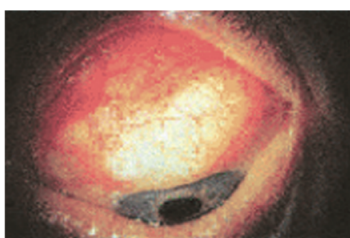

V2: Mild vascularity

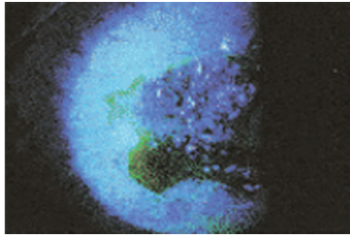

S1: Multiple pinpoint leaks

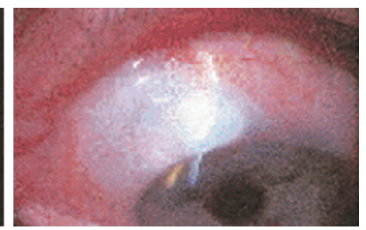

H3: High bleb

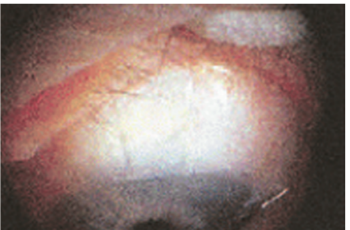

E3: 4 or $>$ clock hours

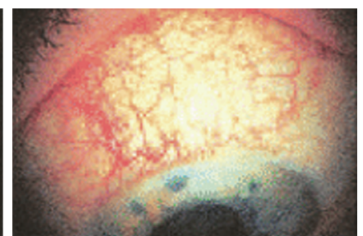

V3: Moderate vascularity

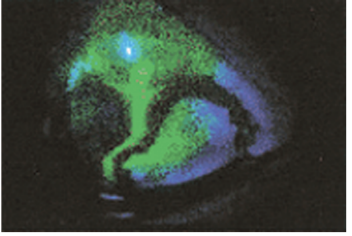

S2: Streaming leak (within $5 \mathrm{sec}$ )

Fig. 2: Indiana Bleb Appearance Grading Scale reference images ${ }^{15}$

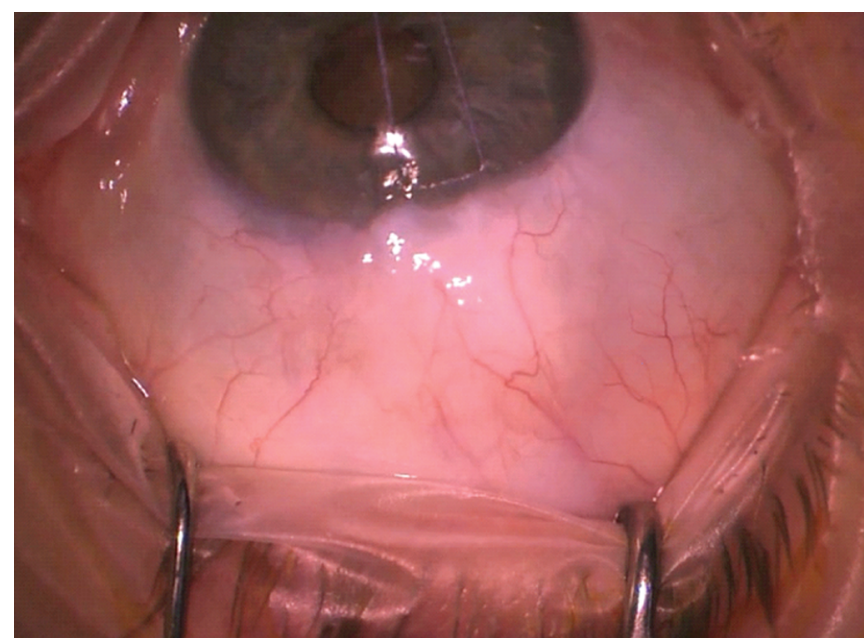

Fig. 3: Wide exposure of over-filtering bleb with 7-0 vicryl stay suture

course. Evaluation occurred at day 1, week 1, 4 weeks, and 3 months postoperatively, before resuming a normal glaucoma-monitoring schedule.

\section{Results}

Four patients with six eyes were included in the study. The mean age of the patients was 66.25 years (range: $64-67$ years). All were female

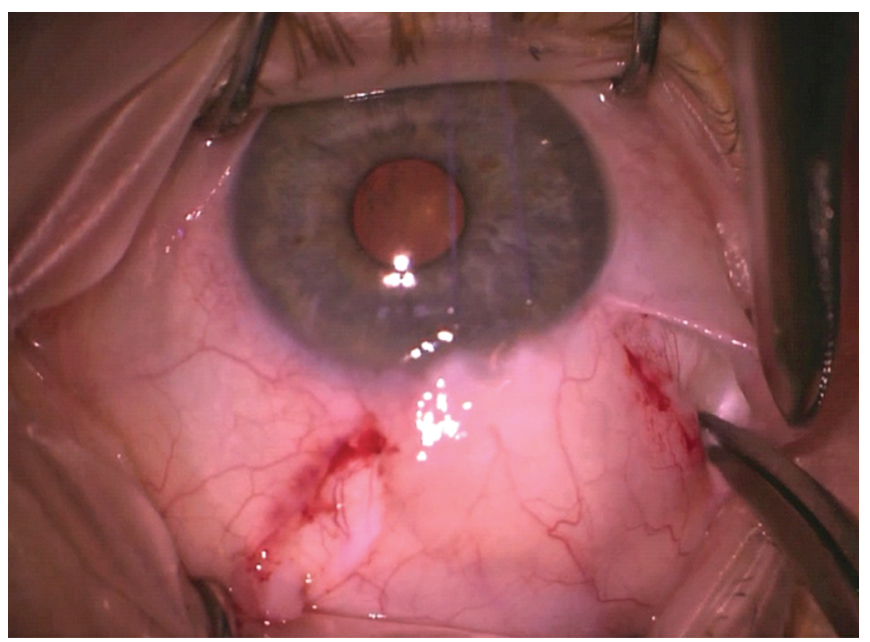

Fig. 4: Radial cutdown with Westcott scissors down to sclera the highest visible portion of the bulbar conjunctiva. Undermining of the conjunctiva was performed to $2 \mathrm{~mm}$ beyond the cut edge away from the bleb

with primary open-angle glaucoma. All were of Caucasian descent. All patients had undergone the same procedure, trabeculectomy enhanced with 5-FU. The mean duration after trabeculectomy to intervention was 18.5 months (range: 6-26 months).

The preoperative characteristics and outcomes are summarized in Table 1. All patients reported a significant reduction in their ocular 


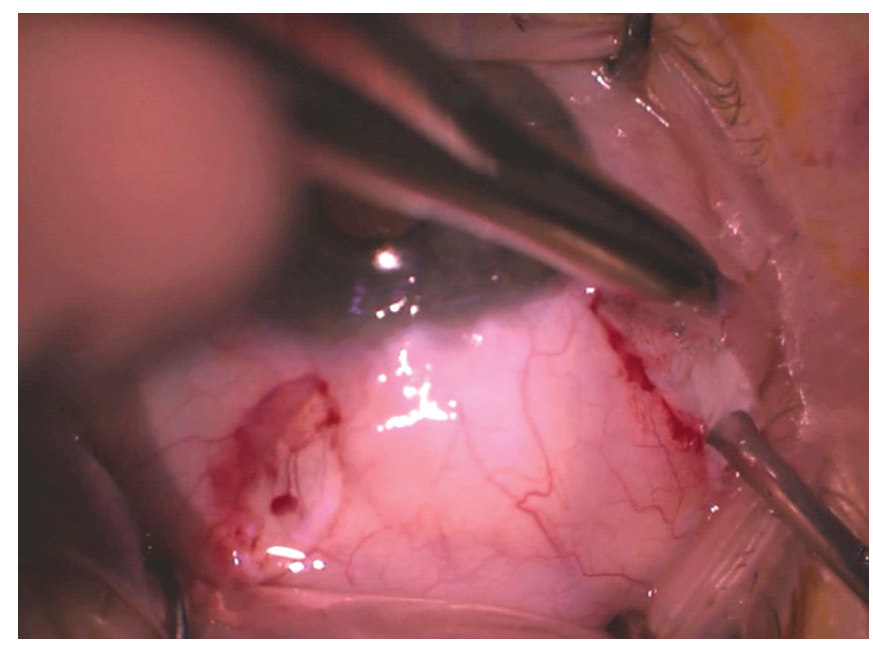

Fig. 5: Introduction of ARTISS glue in its separate constituents (more viscous component first) into both sides of the wound

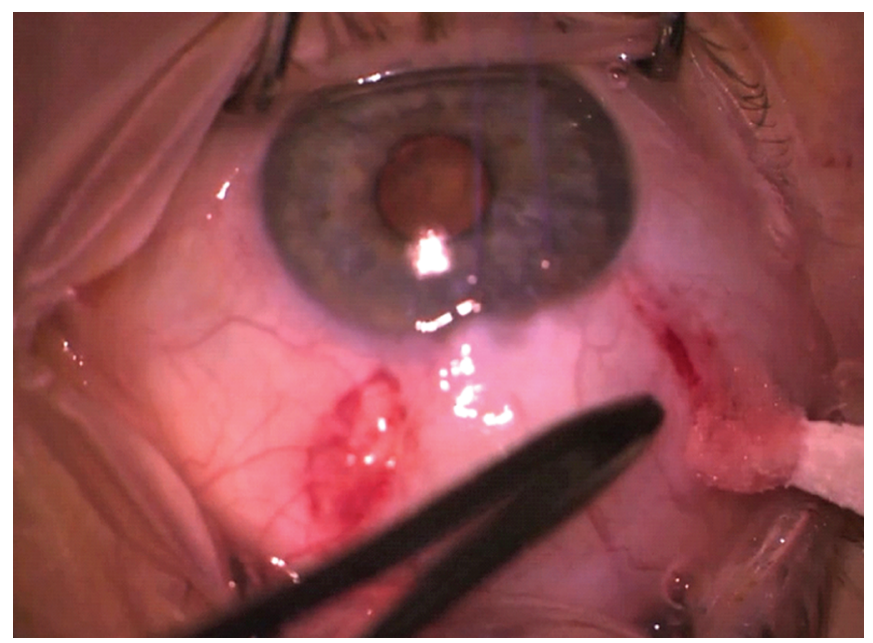

Fig. 7: Compression of glued conjunctiva with spear

Table 1: Patient preoperative characteristic and outcomes

\begin{tabular}{lll}
\hline & Preoperative (range) & $\begin{array}{l}6 \text { months } \\
\text { postoperative } \\
\text { (range) }\end{array}$ \\
\hline $\begin{array}{l}\text { Bleb discomfort score } \\
\text { (subjective 0-10 higher }\end{array}$ & $7(6-8)$ & $3 \pm 0.4(p<0.01)$ \\
number is worse) & $11.25(10-13)$ & $12(10-14)$ \\
IOP (mm Hg) & $6 / 7.125(6 / 6-6 / 7.5)$ & $6 / 7.125(6 / 6-6 / 7.5)$ \\
Visual acuity (Snellen) & $0.65(0.5-0.8)$ & $0.65(0.5-0.8)$ \\
Cup to disk ratio & $-3.2 \pm 0.5 \mathrm{~dB}$ & $-3.2 \pm 0.5 \mathrm{~dB}$ \\
Visual field (logMar) & - & 0 \\
Bleb failure & 0 & 0 \\
Number of medications & 0 & \\
\hline
\end{tabular}

discomfort, reducing from a mean subjective pain score of 7 to 3 ( $p<0.01$, standard deviation: $0.57 ;$ Fig. 8 ). This effect was noted as early as 4 weeks postoperatively.

There was no change in IOP at 6 months post-intervention, and visual acuity was preserved at preoperative levels. There was no impact on visual field mean defect nor cup-to-disk (C:D) ratios.

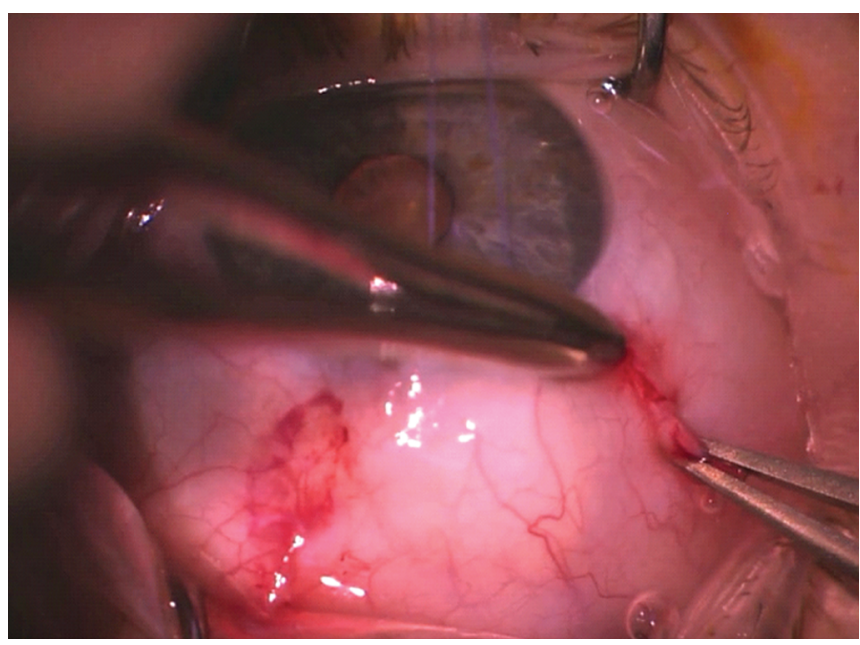

Fig. 6: Reapposition of cut conjunctival edge with Moorefield's forceps with direct adhesion of the two incised edges along their length

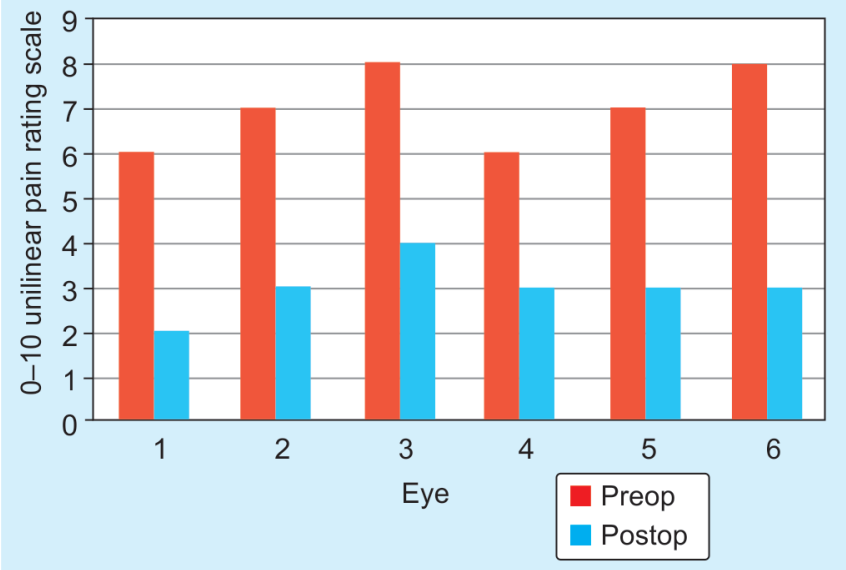

Fig. 8: Graph of pre- and postoperative pain scores for the individual eyes included in the study

Objective measures of bleb morphology postprocedure showed a significant reduction in bleb size, no intrapalpebral bleb extension, no signs of inflammation, and no signs of failure. The mean extension of the bleb was reduced from grade 3 to grade 2 . The bleb height had a minimal reduction from mean grade 2 to 1.9, and vascularity was unchanged at mean grade 1.8 . All blebs were Seidel negative pre- and postoperatively (Fig. 9). There were no reported intra- or postoperative complications including infection, ptosis, or bleb leak.

\section{Discussion}

Bleb dysesthesia is a relatively common problem affecting bleb-forming procedures, in particular trabeculectomy. The incidence has increased in recent years with augmentation of the procedure with antimetabolites such as 5-FU and MMC. This has led to a larger number of patients who are dissatisfied with their treatments despite enhanced IOP control and reduction of glaucoma progression. There is a significant psychological burden associated with trabeculectomy surgery in some subjects due to suffering from constant ocular irritation. The procedure detailed in this report is a novel technique that has been derived 


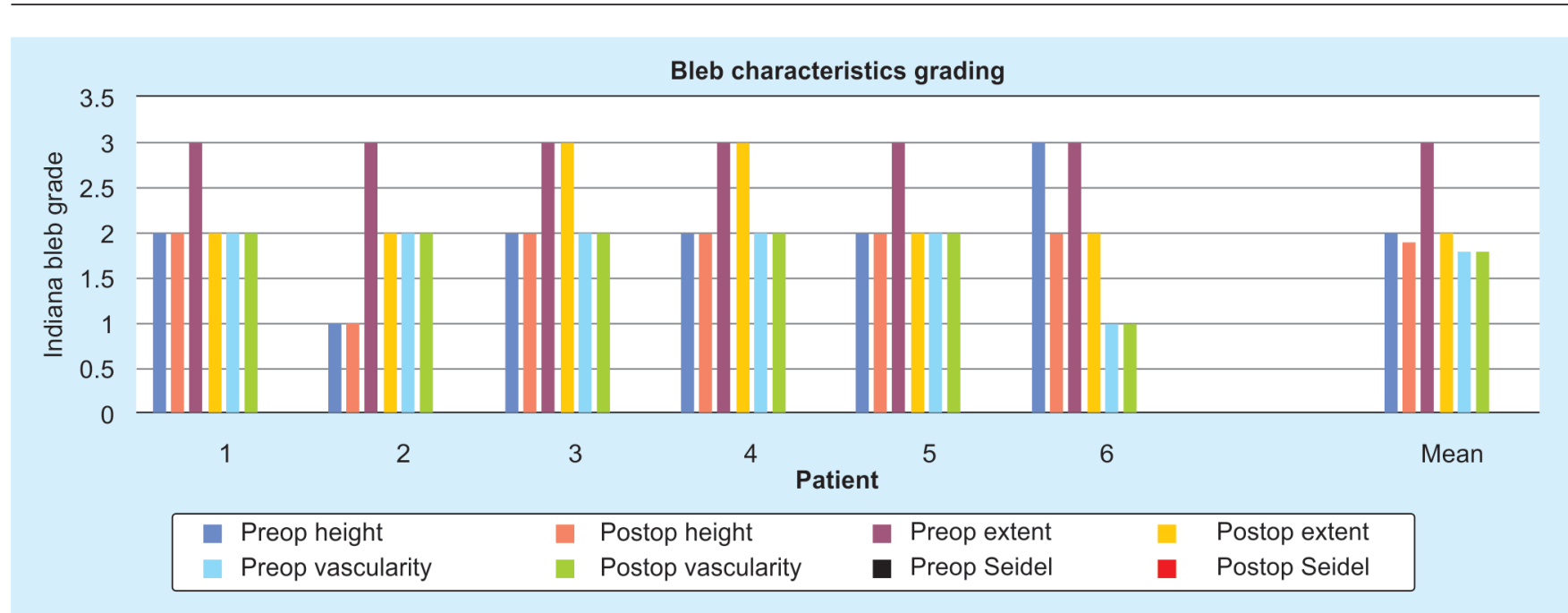

Fig. 9: Graph of the pre- and postoperative bleb characteristics for the individual eyes included in the study (NB: no eyes were Seidel positive pre- or postoperatively)

from the experience of other conjunctival interventions, such as pterygium surgery, with the intention of reducing bleb dysesthesia associated with intrapalpebral bleb extension without unduly adding to the physical or psychological impact of a surgical intervention. ${ }^{4,5,7,9}$

This study is a small pilot series with a relatively short duration of follow-up, as such a definitive statement on long-term safety or efficacy is not possible. This study does, however, demonstrate that the intraoperative surgical technique is not complex, utilizes minimal instrumentation, and can be easily learnt. The procedure time is short, and although this series was performed in the operating theater, it would be possible to undertake in a minor operating, or even in an outpatient setting, depending on comfort and experience. Importantly, there were no intraoperative complications, and the early postoperative course has been unremarkable in this series. It is anticipated that the technique should be safe long-term. Careful preoperative evaluation and surgical technique should obviate most potential problems.

The described technique is designed to limit the bleb size and ensure that it remains under the upper lid. The function of a bleb is most easily compared with a sponge that absorbs the drained aqueous into small pockets with tissue bridges, rather than a large single lake of aqueous. Incising the bleb edge, therefore, should not cause a complete loss of the bleb. While the bleb is reduced in size, it suffered no loss of function as can be noted with no change in IOP. Additionally, there was no contribution to progression of glaucoma over 6 months, as evidenced by no change in visual field or C:D parameters. This was not unexpected, as previous studies have shown that although blebs can be reduced in size, their functionality often remains. As previously stated though, 6 months is a very short time frame for glaucoma.

An important aim of the technique was to reduce the subjective experience of bleb dysesthesia in a dissatisfied group of patients. The series, although small, demonstrates a significant improvement in self-reported pain scores, which is consistent with the literature when barrier techniques are employed. In this cohort, pain scores were reduced in the early postoperative phase, and remained so at 6 months, suggesting that the technique is consistent and effective in reducing bleb dysesthesia, with the added benefit of minimal surgical disruption at early postoperative time points.
The perceived postoperative comfort is largely attributed to the use of fibrin glue rather than sutures. Fibrin glue makes this procedure far quicker than techniques relying on suturing or grafting. It also enables a very even adherence of the revised conjunctiva to the underlying sclera and the easy creation of a watertight seal. The risk of buttonhole formation is minimal and easily treated with the glue itself. There is minimal inflammation as the fibrin glue is reabsorbed, as opposed to dissolvable sutures, and there is no need for removal of sutures, such as in the nonabsorbable type. Fibrin glue has been proven to be safe in human trials and has been used in ophthalmology, particularly in pterygium and tube procedures, for a number of years with minimal adverse events. The improved comfort afforded by this technique, minimal inflammation, and the fact that there is no need for further procedures such as removal of sutures are particularly important in improving patient satisfaction. ${ }^{13}$

Procedures for bleb dysesthesia are often performed in patients who are already unhappy and, in general, unwilling to undergo further procedures. The short duration of the procedure in addition to the relatively painless technique employed makes this a considered choice for this cohort. Further study is warranted, and as longer follow-up and a larger series is accumulated, confidence in the procedure will rise and, as a consequence, will lead to a significant improvement in the quality of life for those affected by bleb dysesthesia.

\section{Conclusion}

There are many reported ways of managing bleb dysesthesia due to intrapalpebral bleb extension without hypotony. Unfortunately, a lot of described techniques are not well accepted by an already dissatisfied group of patients. The key to a successful treatment is to restore patients' comfort while maintaining a fully functioning bleb. Simple techniques are often effective at achieving this. This small pilot series demonstrated that patients suffering from bleb dysesthesia due to intrapalpebral bleb extension can be successfully treated with a novel surgical approach by combining targeted conjunctival dissection down to sclera with fibrin tissue glue closure. This study demonstrated no significant effect on trabeculectomy bleb function and did not contribute to glaucoma progression over a 6-month period. Importantly, the technique was well accepted 
by subjects, there was a significant reduction in self-reported discomfort, and no new surgical complications were noted.

\section{Clinical Significance}

Bleb dysesthesia following antifibrotic-enhanced trabeculectomy is common, resulting in a significant decrease in quality of life for those affected. This study demonstrates a novel surgical approach to treat patients with this condition, in a safe, simple, but effective manner. The technique is easily learnt and can be employed in an outpatient setting. The technique is readily accepted by patients in group who are often reticent to undergo further intervention, and most importantly, it does not compromise bleb function nor does it destabilize glaucoma control.

\section{References}

1. Beatty S, Potamitis T, Kheterpal S, et al. Trabeculectomy augmented with mitomycin C application under the scleral flap. Br J Ophthalmol 1998;82(4):397. DOI: 10.1136/bjo.82.4.397.

2. Wilkins M, Indar A, Wormald R. Intraoperative mitomycin C for glaucoma surgery. Cochrane Database Syst Rev 2005(4). DOI: 10.1002/14651858.CD002897.pub2.

3. Cabourne E, Clarke JCK, Schlottmann PG, et al. Mitomycin C versus 5 -fluorouracil for wound healing in glaucoma surgery. Cochrane Database Syst Rev 2015;(11):CD006259. DOI: 10.1002/14651858. CD006259.pub2.

4. Budenz DL, Hoffman K, Zacchei A. Glaucoma filtering bleb dysesthesia. Am J Ophthalmol 2001;131(5):626-630. DOI: 10.1016/ s0002-9394(00)00901-6.
5. Goyal N, Sun Choi H, Brown S. Treating bleb dysaesthesia. Glaucoma today 2011;1:33-35.

6. Shonibare O. Management of intractable bleb dysesthesia. Eye News 2016;23(2):37-39.

7. Catoira-Boyle $Y$, Wudunn D, Cantor L. Revision of dysfunctional filtering blebs by conjunctival advancement with bleb preservation. Am J Ophthalmol 2000;130(5):574-579. DOI: 10.1016/S00029394(00)00653-X.

8. Wadhwani RA, Bellows AR, Hutchinson BT. Surgical repair of leaking filtering blebs. Ophthalmology 2000;107(9):1681-1687. DOI: 10.1016/ s0161-6420(00)00282-7.

9. Begum $\mathrm{S}$, Bobat $\mathrm{H}$, Kirwan J. Bleb compression and autologous blood for relief of bleb dysaesthesia. Does it work? Clin Experiment Ophthalmol 2016;44(6):528-549.

10. Geyer O.Management of large, leaking, and inadvertent filtering blebs with the neodymium: YAG laser. Ophthalmology 1998;105(6):983-987. DOI: 10.1016/S0161-6420(98)96023-7.

11. Anis S, Ritch R, Shihadeh W, et al. Surgical reduction of symptomatic, circumferential, filtering blebs. Arch Ophthalmol 2006;124(6):890894. DOI: 10.1001/archopht.124.6.890.

12. Tannenbaum DP, Hoffman D, Greaney MJ, et al. Outcomes of bleb excision and conjunctival advancement for leaking or hypotonous eyes after glaucoma filtering surgery. Br J Ophthalmol 2004;88(1):99. DOI: 10.1136/bjo.88.1.99.

13. Panda A, Kumar S, Kumar A, et al. Fibrin glue in ophthalmology. Indian J Ophthalmol 2009;57(5):371-379. DOI: 10.4103/0301-4738.55079.

14. Cirino E, Pain Scale: Healthline Media; 2017 [Available from: https:// www.healthline.com/health/pain-scale.

15. Cantor L, Mantravadi A, Wudunn D, et al. Morphologic classification of filtering blebs after glaucoma filtration surgery: The Indiana bleb appearance grading scale. J Glaucoma 2003;12(3):266-271. DOI: 10.1097/00061198-200306000-00015. 